\title{
Contributing Reviewers in 2015
}

J Pediatr Intensive Care 2016;5:i.

Journal of Pediatric Intensive Care wishes to recognize those who contributed as an expert peer reviewer of submitted scientific papers in 2015.

Thank you for your contributions to Journal of Pediatric Intensive Care in 2015.

Marcelo Luiz Abramczyk

Tarek Alhamad

Eman S. Al-Khadra

Collin R. Anderson

Vinod K. Bansal

Tellen D. Bennett

Laura A. Bonouvrié

Matthew S. Braga

Thomas V. Brogan

Stefan Bushuven

Ming-Ren Chen

Gulnur Com

Morag Curnow

Işıl Davarcı

Angela De Cunto

Suelen Rosa de Oliveira

Eugene Michael Dempsey

Alan C. Fenton

Sotirios Fouzas

Soren Gantt

James Gerard

Pauline Catherine Gillan

Frank Dunbar Groves

Lokesh Guglani

Josée Guindon

Eric J. Haas

Grenville Peter Hadley
Andrei Harabor

Moustafa Abdelaal Hegazi

Edgar Debray Hernández-Álvarez

Robert P. Hoffman

Kam-Lun Ellis Hon

Noriaki lino

Pran M. Kar

Jost Kaufmann

Anant Khositseth

Jyrson Guilherme Klamt

Kathy Kortes-Miller

Bhanumathi Lakshminarayanan

Dan-Di Li

Jiu-Jun Li

Nathalie Maitre

M Loredana Marcovecchio

Abraham Markin

Giuseppe A. Marraro

Colin P. Marsland

Charles McKay Jr

Kelly A. Michienzi

Alexander J. Mittnacht

Ronald P. Mlcak

Frances M. Nadel

Komal J. Narwaney

Sonali S. Patel

Kurt David Piggott
Joaquim M.B. Pinheiro

Ajna Poulsen

Caroline M. Quill

Ahmad Usaid Qureshi

Satish K. Rajagopal

Margie A. Ream

Antonio Rodríguez-Núñez

Ewa Romejko-Wolniewicz

Jonathan Roth

Mohamed Saleem

Achim Schmitz

Hazel Ann Smith

Michael Spaeder

Carlotta Spagnoli

Uma Srivastava

Astrid G. Stucke

Lisa Sun

Sadeka Tamanna

Tiago Neves Veras

Asse Kouadio Vincent

Yehezkel Waisman

Jennifer Weil

Fu-Shan Xue

Jill Zalieckas

Jianhui Zhang 